\title{
Update on quetiapine in the treatment of bipolar disorder: results from the BOLDER studies
}

\author{
Prashant Gajwani' \\ David J Muzina ${ }^{2}$ \\ David E Kemp 3 \\ Keming Gao' \\ Joseph R Calabrese' \\ 'Case Western Reserve University \\ (CWRU) School of Medicine, \\ ${ }^{2}$ Cleveland Clinic Lerner College of \\ Medicine of CWRU, ${ }^{3}$ Case Western \\ Reserve University, Cleveland $\mathrm{OH}$, \\ USA
}

\begin{abstract}
The essential features of bipolar affective disorder involve the cyclical occurrence of high (manic or hypomanic episodes) and low mood states. Depressive episodes in both bipolar I and II disorder are more numerous and last for longer duration than either manic or hypomanic episodes. In addition depressive episodes are associated with higher morbidity and mortality. While multiple agents, including all 5 atypical antipsychotics, have demonstrated efficacy and earned US FDA indication for manic phase of bipolar illness, the acute treatment of bipolar depression is less well-studied. The first treatment approved by the US FDA for acute bipolar depression was the combination of the atypical antipsychotic olanzapine and the antidepressant fluoxetine. Recently, quetiapine monotherapy has demonstrated efficacy in the treatment of depressive episodes associated with both bipolar I and II disorder and has earned US FDA indication for the same.
\end{abstract}

Keywords: bipolar disorder, quetiapine, BOLDER studies

\section{Introduction}

Bipolar disorder is estimated to affect 3\%-7\% of population (Angst 1998; Kessler et al 2005). Numerous US FDA-approved treatment options exist for the treatment of manic phase of bipolar disorder, including lithium, valproic acid and all 5 atypical antipsychotics - olanzapine, risperidone, quetiapine, ziprasidone and aripiprazole (Gajwani et al 2006).

The burden of bipolar disorder arises mostly from the depressed phase for both bipolar I and II disorder. Patients with bipolar I disorder experience depressive symptoms for 3 times longer compared with manic periods and report longer recovery times for depressed phases compared with manic phases of the illness (Hlastala et al 1997; Judd et al 2002). Patients with bipolar II disorder experience even greater duration and severity of depressive symptoms as compared to hypomanic mood disturbances (Judd et al 2003). Bipolar disorder-related morbidity and mortality can be due to suicide attempts and completed suicide. The depressed phase of bipolar disorder is associated with an increased risk of suicide compared with the manic phase of the illness (Leverich et al 2003; Post et al 2003). Bipolar depression is also associated with higher impairments in the psychosocial spheres of family, social and occupational functioning compared with bipolar mania (Calabrese et al 2004). Even in the absence of major depressive episode, subsyndromal depressive symptoms (presence of 2 or more depressive symptoms) in bipolar disorder are associated with some disability or functional impairment (Altshuler et al 2002). Because of the predominance of depressive symptomalogy, the greater time spent in depressive episodes and higher frequency of depressive episodes often leads to a misdiagnosis of bipolar disorder as major depressive disorder. This can lead to patients receiving inadequate or inappropriate treatment such as antidepressant monotherapy. Despite the prevalence and impact of depression in bipolar disorder, there are currently only 2 FDA-approved 
agents for its management compared with multiple approved agents for management of bipolar mania.

Historically, clinicians have commonly employed combination pharmacotherapies for bipolar depression, usually an antidepressant and a mood stabilizer. Most of the treatment guidelines limit the exposure of antidepressants to a few months during acute depressive episodes due to concerns that it may precipitate treatment emergent mania or cycle acceleration. Tricyclic antidepressants and monoamine oxidase inhibitors confer the highest risk for induction of mania whereas rates for induction of mania with selective serotonin re-uptake inhibitors (SSRIs) are similar to placebo (Goodwin and Jamison 1990; Himmelhock et al 1991; Peet 1994).

The purpose of this paper is to review recent evidence in support of quetiapine monotherapy for the acute treatment of depressive episodes associated with bipolar disorder, as well as the data related to safety and tolerability of this atypical antipsychotic medication in bipolar depressed patients.

\section{Study design}

Quetiapine monotherapy was evaluated for efficacy and tolerability for patients with BipOlar DEpRession (BOLDER) in two separate trials (Calabrese et al 2005; Thase et al 2006). Both BOLDER I and II trials were identical in design. A double-blind, randomized, fixed-dose, placebo-controlled, parallel-group monotherapy design was employed for each study. BOLDER I was conducted at 39 centers in the US between September 2002 and October 2003. BOLDER II was conducted at 41 centers in the US between June 2004 and August 2005. There were 21 centers in common between the two trials. Compared with prescription of quetiapine in bipolar mania trials where patients were hospitalized and received quetiapine twice a day, the BOLDER trials enrolled outpatients and quetiapine was administered in once-a-day dosing (Gajwani et al 2006). These trials were designed to examine efficacy of 2 fixed doses of quetiapine, $300 \mathrm{mg}$ and $600 \mathrm{mg}$ per day, compared with placebo, in patients with moderate to severe bipolar depression. The structured clinical interview for Diagnostic and Statistical Manual of Mental Disorders, fourth edition (DSM-IV) was used to confirm diagnosis. Prior to study initiation, all raters were trained and certified. As much as possible, each patient's rating was to be performed by the same rater using throughout the study.

Inclusion criteria allowed outpatients (aged 18-65 years) who met DSM IV criteria for bipolar I or II disorder and were experiencing a major depressive episode. Patients with rapid-cycling bipolar disorder were also included. Patients were required to have Hamilton Depression Rating Scale
(Hamilton 1960) (HAM-D) 17-item score $\geq 20$, a Hamilton Depression Scale item 1 score $\geq 2$ and Young Mania Rating Scale (Young et al 1978) score $\leq 12$ at both screening and randomization. To minimize potential rating inflation of the screening and randomization scores, inclusion criteria were based on initial HAM-D score while the primary efficacy measure during the study was based on Montgomery-Åsberg Depression Rating Scale scores (MADRS) (Montgomery and Åsberg 1979). The primary efficacy variable was the change in MADRS total score from baseline to week 8 . The following secondary outcomes were also measured: the proportion of patients achieving response $(\geq 50 \%$ reduction in MADRS score from baseline), the proportion of patients who achieved remission (MADRS $\leq 12$ ) and analysis of individual MADRS items.

Exclusion criteria included (1) diagnosis of an Axis I disorder other than bipolar disorder that was the primary focus of treatment within last 6 months, (2) a current episode of depression that had lasted more than 12 months or less than 4 weeks in duration, (3) failure to respond to 2 adequate (6 weeks) trial of more than 2 classes of antidepressants during the current episode, and (4) a current (within last 12 months of screening) diagnosis of substance dependence or abuse (except nicotine). Patients with significant medical illnesses or patients who currently posed serious risk of suicide or homicide were also excluded.

After obtaining written informed consent, patients were randomly assigned to either of the three treatment arms - quetiapine $300 \mathrm{mg}$, quetiapine $600 \mathrm{mg}$ or placebo (Table 1). Random assignment was stratified according to bipolar type (I or II) to ensure 2:1 ratio for bipolar diagnosis. Lorazepam (1-3 mg/day) and zolpidem tartarate (5-10 mg at bedtime) were permitted during the first 3 weeks of randomization but were withheld for 8 hours prior to psychiatric assessment. Enrolled patients underwent a washout period for 7-28 days wherein any prior medications were tapered off and were then treated for 8 weeks in double-blinded fashion. Quetiapine was initiated at $50 \mathrm{mg} /$ day and titrated to achieve $300 \mathrm{mg}$ /day by day 4 or $600 \mathrm{mg} /$ day by day 7 .

\section{Results}

During the BOLDER I trial, a total of 838 patients were screened and 542 patients with bipolar I $(n=360)$ or II ( $\mathrm{n}=182$ ) were randomly assigned to receive quetiapine $600 \mathrm{mg} /$ day $(\mathrm{n}=180)$, quetiapine $300 \mathrm{mg} /$ day $(\mathrm{n}=181)$, or placebo $(\mathrm{n}=180)$. Of the randomized patients, 511 received at least one post baseline assessment and were analyzed for efficacy in the intent to treat analysis. There 
Table I Comparing the BOLDER studies

\begin{tabular}{|c|c|c|c|c|c|c|}
\hline & \multicolumn{3}{|c|}{ BOLDER I } & \multicolumn{3}{|c|}{ BOLDER II } \\
\hline & Placebo & Seroquel-300 & Seroquel-600 & Placebo & Seroquel-300 & Seroquel-600 \\
\hline $\mathrm{N}$ & 181 & 181 & 180 & 168 & 172 & 169 \\
\hline \multicolumn{7}{|l|}{ DSM-IV diagnosis } \\
\hline BP I (\%) & 66.3 & 67.4 & 67.1 & 68.3 & 67.1 & 66.9 \\
\hline BP II (\%) & 33.7 & 32.6 & 32.9 & 31.7 & 32.9 & 33.1 \\
\hline $\mathrm{RCBD}$ & 20.7 & 24.4 & 18.2 & 32.9 & 28.4 & 30.5 \\
\hline mean age & 38.3 & 36.6 & 37.3 & 37.7 & 37.2 & 38.2 \\
\hline \multicolumn{7}{|l|}{$\operatorname{sex}(\%)$} \\
\hline male & 37.9 & 45.9 & 41.8 & 39.8 & 44.5 & 45 \\
\hline female & 62.1 & 54.1 & 58.2 & 60.2 & 55.5 & 55 \\
\hline \multicolumn{7}{|l|}{ baseline scores } \\
\hline MADRS & 30.6 & 30.4 & 30.3 & 29.6 & 31.1 & 29.9 \\
\hline HAM-D & 24.6 & 24.5 & 24.7 & 24.3 & 24.9 & 24.3 \\
\hline CGI & 4.4 & 4.4 & 4.5 & 4.5 & 4.6 & 4.4 \\
\hline \multicolumn{7}{|c|}{ Mean change in score } \\
\hline MADRS & -10.26 & -16.39 & -16.73 & -11.93 & -16.94 & -16 \\
\hline HAM-D & -8.54 & -13.38 & -13.84 & -9.92 & -13.81 & -12.97 \\
\hline CGI & 2.97 & 2.27 & 2.37 & 2.88 & 2.28 & 2.29 \\
\hline Response rates & $36.10 \%$ & $58 \%$ & $58 \%$ & $44.70 \%$ & $60.00 \%$ & $58.30 \%$ \\
\hline Remission rates & $28.40 \%$ & $52.90 \%$ & $52.90 \%$ & $37.30 \%$ & $51.60 \%$ & $52.30 \%$ \\
\hline Effect size in BP I & & 0.91 & 1.09 & & 0.67 & 0.51 \\
\hline Effect size in BP II & & 0.28 & 0.39 & & 0.56 & 0.64 \\
\hline
\end{tabular}

Abbreviations: BP, bipolar; CGI, Clinical Global Impression; DSM-IV, Diagnostic and Statistical Manual of Mental Disorders, fourth edition; HAM-D, Hamilton depression rating scale; MADRS, Montgomery-Åsberg Depression Rating Scale scores; RCBD, rapid cycling bipolar disorder.

were no significant differences in baseline characteristics. The mean age was approximately 37 years and $58.2 \%$ of the patients were women. Patients' baseline MADRS scale scores reflected moderate to severe depression $(\sim 30)$ (Muller et al 2003). The proportion of patients completing the study was $54 \%$ in the quetiapine $600 \mathrm{mg} /$ day group, $67 \%$ in the quetiapine $300 \mathrm{mg} /$ day group and $59 \%$ in the placebo group. The most common reason for withdrawal from the study was adverse events in the quetiapine group $(26.1 \%$ for 600 $\mathrm{mg} /$ day and $16 \%$ for $300 \mathrm{mg} /$ day) and lack of efficacy in the placebo group (13.3\%).

Quetiapine at a daily dose of either $300 \mathrm{mg}$ or $600 \mathrm{mg}$ demonstrated significant improvement in the MADRS total scale scores as early as week 1 and at all times thereafter. The mean change in the MADRS scale scores from baseline to last assessment was -16.73 in the quetiapine 600 $\mathrm{mg}$ /day group, -16.39 in the quetiapine $300 \mathrm{mg}$ /day group compared -10.26 in the placebo group. At final assessment, approximately $58 \%$ patients met response criteria in the quetiapine groups compared with $36.1 \%$ in the placebo group. Remission criteria were met by $52.9 \%$ of patients in both quetiapine groups compared with placebo group's $28.4 \%$. On individual MADRS item factor analysis, 9 out of 10 depression scale items were significantly improved in the quetiapine $600 \mathrm{mg}$ /day group and 8 out of 10 in the quetiapine $300 \mathrm{mg}$ /day group. Both doses of quetiapine were more effective than placebo in reducing suicidal thoughts at final assessment. Statistically superior improvement was seen from baseline in MADRS total score irrespective of the type of illness (bipolar I or II) or presence of rapid cycling compared with placebo. Quetiapine-treated patients also demonstrated statistically significant improvement on the Clinical Global Impression (CGI) improvement scale (much or very much improved was $55.9 \%$ for the quetiapine 600 $\mathrm{mg}$ /day group, $64 \%$ for the quetiapine $300 \mathrm{mg} /$ day group compared with $34.3 \%$ on placebo.

In the BOLDER II trial, a total of 788 patients were screened and 509 patients with bipolar I $(\mathrm{n}=338)$ or bipolar II $(\mathrm{n}=171)$ disorder were randomly assigned to receive quetiapine $600 \mathrm{mg} /$ day $(\mathrm{n}=169)$, quetiapine $300 \mathrm{mg} /$ day $(n=172)$ or placebo $(n=168)$. Of the randomized patients, 467 received at least one post-baseline assessment and were analyzed for efficacy in the intent-to-treat analysis. There were no significant differences in baseline characteristics. The mean age was approximately 37 years and 57\% of the patients were women. Patients baseline MADRS scale scores reflected moderate to severe depression (ranged from 29.6-31.1) (Muller et al 2003). The proportion of patients completing the study was $53.3 \%$ in the quetiapine $600 \mathrm{mg} /$ day group, $58.7 \%$ in the quetiapine $300 \mathrm{mg} /$ day group and 
$65.5 \%$ in the placebo group. The most common reason for withdrawal in the quetiapine-treatment groups was adverse events versus lack of efficacy in the placebo group.

Quetiapine at either dose led to significantly greater improvement in MADRS total score at week 1 and at all times thereafter compared with placebo. The mean change in the MADRS scale scores from baseline to last assessment was -16.00 in the quetiapine $600 \mathrm{mg}$ /day group and -16.94 in the quetiapine $300 \mathrm{mg}$ /day group compared with -11.93 in the placebo group. At final assessment, approximately $58.3 \%-60 \%$ patients met response criteria in the quetiapine groups compared with $44.7 \%$ in the placebo group. Remission criteria were met by $51.6 \%-52.3 \%$ of patients in both quetiapine groups compared the placebo group's $37.3 \%$. On MADRS item analysis in this study, the majority of individual depression scale items significantly improved with both $300 \mathrm{mg}$ and $600 \mathrm{mg}$ quetiapine treatment. Both doses of quetiapine were more effective than placebo in reducing suicidal thoughts at final assessment. Statistically superior improvement was seen from baseline in MADRS total score irrespective of the type of illness (bipolar I or II) or presence of rapid cycling as compared to placebo. Quetiapine-treated patients also demonstrated statistically significant improvement on the CGI severity and improvement scale ("much improved" or "very much improved" was reported in $60 \%$ of the quetiapine 600 $\mathrm{mg} /$ day group, $61.3 \%$ of the quetiapine $300 \mathrm{mg}$ /day group and only $38.5 \%$ of the placebo).

BOLDER I reported the effect size in bipolar I depression to be 1.09 for those patients assigned to the $600 \mathrm{mg}$ /day group and 0.91 for those taking $300 \mathrm{mg}$ /day of quetiapine. The effect size in the bipolar II subgroup was 0.39 in the 600 $\mathrm{mg}$ /day group and 0.28 in the $300 \mathrm{mg}$ /day group. BOLDER II reported effect size in bipolar I subgroup to be 0.51 for the $600 \mathrm{mg}$ /day group and 0.67 for the $300 \mathrm{mg} /$ day group. The reported effect size in the bipolar II subgroup was 0.64 for patients receiving $600 \mathrm{mg} /$ day and 0.56 for patients receiving $300 \mathrm{mg} /$ day of quetiapine.

\section{Safety and tolerability}

During the BOLDER I trial, 2 patients attempted suicide, one in each of the quetiapine groups, but no suicides or deaths occurred during the study. The overall rate of study discontinuation due to adverse events was $26.1 \%(n=47)$ in the $600 \mathrm{mg} /$ day group, $16.0 \%(\mathrm{n}=29)$ in the $300 \mathrm{mg} /$ day group and $8.8 \%(n=16)$ in the placebo group. The incidence of treatment-emergent mania was low in the quetiapine groups compared with placebo $(2.2 \%$ with $600 \mathrm{mg} /$ day, $3.9 \%$ with
$300 \mathrm{mg} /$ day and $3.9 \%$ with placebo). Extrapyramidal adverse events were present in $8.9 \%$ of the $600 \mathrm{mg}$ /day group, $6.7 \%$ of the $300 \mathrm{mg} /$ day group and $2.2 \%$ of the placebo group. The rate of discontinuation in the quetiapine group due to extrapyramidal symptoms was low $(1.1 \%-2.8 \%)$. Reported weight gain was as follows: $0.2 \mathrm{~kg}$ in the placebo group, $1.0 \mathrm{~kg}$ in the $300 \mathrm{mg} /$ day group, $1.6 \mathrm{~kg}$ in the $600 \mathrm{mg} /$ day group. Significant weight gain as defined by weight gain of $\geq 7 \%$ from baseline was reported in $1.7 \%$ in the placebo group, $8.5 \%$ in the $300 \mathrm{mg}$ /day group and $9.0 \%$ in the 600 $\mathrm{mg}$ /day group.

During the BOLDER II study, no deaths occurred. The overall rate of study discontinuation due to adverse events was $11.2 \%(n=19)$ in the $600 \mathrm{mg} /$ day group, $8.1 \%(\mathrm{n}=14)$ in the $300 \mathrm{mg} /$ day group and $1.2 \%(\mathrm{n}=2)$ in the placebo group. The incidence of treatment-emergent mania was higher in the placebo group than in either of the quetiapine groups $(3.6 \%$ with $600 \mathrm{mg} /$ day, 1.8\% with $300 \mathrm{mg} /$ day and $6.6 \%$ with placebo). Extrapyramidal adverse events were present in $10.1 \%$ of the $600 \mathrm{mg} /$ day group, $12.3 \%$ of the 300 $\mathrm{mg} /$ day group and $6.6 \%$ of the placebo group. The rate of discontinuation in the quetiapine group due to extrapyramidal symptoms was low ( $0.6 \%$ in the $600 \mathrm{mg}$ /day group). Reported weight gain was as follows: $0.3 \mathrm{~kg}$ in the placebo group, 1.4 $\mathrm{kg}$ in the $300 \mathrm{mg} /$ day group, $1.3 \mathrm{~kg}$ in the $600 \mathrm{mg} /$ day group. Significant weight gain as defined by weight gain of $\geq 7 \%$ from baseline was reported in $2.8 \%$ in placebo group, $3.9 \%$ in the $300 \mathrm{mg} /$ day group and $8.6 \%$ in the $600 \mathrm{mg} /$ day group. There was no significant change in the fasting blood glucose level from baseline to final assessment.

During both BOLDER I and II trials, no patient withdrew from the study due to weight gain. There was no significant change in the fasting blood glucose level from baseline to final assessment with quetiapine or placebo treatment and the mean fasting glucose level for all groups remained within the normal range. Also, no clinically important differences between groups were reported in both BOLDER studies in the mean change from baseline for any vital signs, electrocardiograms, hematology, or clinical chemistry parameters.

\section{Discussion}

The BOLDER studies were large scale, multi-center, randomized, placebo-controlled trials that examined the efficacy of quetiapine monotherapy in acute bipolar depression. These were the first large-scale, controlled studies published to date that enrolled patients with both bipolar I and II disorder and included patients with rapid cycling bipolar disorder. 
Based on the results, quetiapine has demonstrated significant antidepressant efficacy in the depressed phase of bipolar disorder. Based on the primary efficacy scale (MADRS), improvement was evident from week 1 and maintained for the duration of the study ( 8 weeks). In the MADRS item analysis, both doses of quetiapine produced a significant and sustained improvement in most of the core symptoms of mood including decrease in suicidal thinking. Similarly, significant improvement was observed in patients with bipolar I and II disorder and in patients with or without rapid cycling. The therapeutic benefit of quetiapine $300 \mathrm{mg}$ /day and $600 \mathrm{mg}$ /day was similar at study end point. The FDA has approved the quetiapine $300 \mathrm{mg}$ /day dose in the treatment of bipolar depression.

The rate of quetiapine induced treatment-emergent mania was low, $2 \%-4 \%$, and on par with the $4 \%-7 \%$ rate observed with placebo. It can be concluded that antidepressant efficacy of quetiapine was not at the expense of treatment-emergent affective switches. A low risk of treatment-emergent affective switches may be one of the strongest advantages offered in the management of bipolar depression. However, it must be noted that in a short treatment trial such as these 8-week depression studies, this placebo-level switch rate applies only to that brief period studied and cannot exclude the possibility of switches later during the course of ongoing treatment. This seems less likely with an agent such as quetiapine which is also known to be and is an approved as an antimanic agent.

Reported side effects were similar in both trials. Most commonly reported side effects included dry mouth, sedation, somnolence, dizziness and constipation. Sedation and somnolence were the commonest cited reason for withdrawal from the study, with most discontinuation occurring in first week. Higher drop out rates were observed in the quetiapine $600 \mathrm{mg}$ /day arm compared with quetiapine $300 \mathrm{mg}$ /day arm. Weight gain was $1.2 \mathrm{~kg}$ in the quetiapine $300 \mathrm{mg}$ arm and $1.5 \mathrm{~kg}$ in the quetiapine $600 \mathrm{mg}$ arm, and no patients withdrew from study due to weight gain. A small increase in fasting plasma glucose level was observed in the quetiapine and placebo arms and no patients developed diabetes. The mean change in Simpson Angus Scale (SAS) and Barnes Akathisia Rating Scale (BARS) was low and generally comparable across the three arms.

Comparing the two studies overall, higher placebo response rates were seen in the BOLDER II trial which resulted in net lower magnitude of response rates for quetiapine versus placebo. The change from baseline MADRS was comparable in both quetiapine groups in both BOLDER
I and II trials. It is possible that physician and patient bias could have artificially elevated the placebo response rates in BOLDER II trial, as results from BOLDER I study were published and widely known at the time the BOLDER II trial was being conducted. BOLDER II compared with BOLDER I demonstrated significant improvement in the bipolar II subpopulation. Comparable efficacy was observed in the subpopulation of patients with rapid-cycling bipolar disorder compared with non-rapid-cycling bipolar disorder.

Limitations of these studies include lack of data on doses other than $300 \mathrm{mg} /$ day or $600 \mathrm{mg}$ /day of quetiapine in bipolar depression. Also it is difficult to comment on efficacy of the $600 \mathrm{mg}$ /day dose for patients failing to respond to quetiapine $300 \mathrm{mg} /$ day. It is also unknown if patients not responding to quetiapine $600 \mathrm{mg}$ /day would benefit from any further dose titration. Patients at higher risk of suicide were excluded from the study which limits the conclusion of data to acutely suicidal bipolar depressed patients. Patients with current alcohol or illicit drug dependence were excluded, hence no conclusion can be drawn about efficacy of quetiapine in dual-diagnosis bipolar disorder.

In a similar double-blind, placebo-controlled, 8-week-long trial, 833 adults with bipolar depression (MADRS score of at least 20) were randomized to receive placebo $(n=377)$; olanzapine, $5-20 \mathrm{mg} /$ day $(\mathrm{n}=370)$; or olanzapine-fluoxetine combinations, 6 and 25, 6 and 50, or 12 and $50 \mathrm{mg} /$ day $(\mathrm{n}=86)$ (Tohen et al 2003). Mean modal drug dose for olanzapine monotherapy was $9.7 \mathrm{mg} /$ day and $7.4 \mathrm{mg}$ /day of olanzapine with $39.3 \mathrm{mg} /$ day of fluoxetine for olanzapine-fluoxetine arm. At week 8, MADRS scores were lower than baseline by $11.9,15.0$ and 18.5 points in the placebo, olanzapine and olanzapine-fluoxetine groups respectively. The therapeutic effect size was 0.32 for olanzapine and 0.68 for olanzapinefluoxetine combination. The response rates were $30.4 \%$ for placebo, 39\% for olanzapine monotherapy and $56.1 \%$ for olanzapine-fluoxetine combination. Olanzapine-fluoxetine was found to be more effective than olanzapine monotherapy and placebo in the treatment of bipolar depression.

The mechanism of action by which quetiapine can effectively treat bipolar depression is unknown. This antipsychotic and antimanic agent, now with reported antidepressant effects, is an antagonist at multiple neurotransmitter receptor sites in the brain, including 5HT1A, 5HT2, D1, D2, histamine H1, alpha-1 and alpha-2. Its dopamine antagonism may be responsible for its antipsychotic and antimanic effects. Quetiapine is metabolized by 4 routes, including hydroxylation, sulfoxidation, oxidation and dealkylation. Dealkylation generates the primary active metabolite of 
the parent compound, nor-quetiapine. Nor-quetiapine has high affinity and is a potent inhibitor of the noradrenergic transporter $(\mathrm{Ki}=35 \mathrm{nM})$, and has partial agonistic activity at the serotonin 5HT1A receptor (Goldstein et al 2007). These findings suggest that quetiapine interacts with 3 principal neuro-transmitter involved in regulation of mood: norepinephrine, serotonin and dopamine. It is likely that these broader neurotransmitter effects, confer antidepressant properties to quetiapine.

Management of bipolar depression is one of the most challenging tasks faced by clinicians and represents one of the greatest unmet needs for our patients. Antidepressants are frequently used with mood stabilizers for treatment of bipolar depression, despite limited evidence and concerns about potential increased risk of change in mood polarity (Goodwin and Jamison 1990; Leverich et al 2006). In a recently published study (Sachs et al 2007), effectiveness of antidepressants with mood stabilizers was compared with placebo with mood stabilizers. The study was conducted by the Systematic Treatment Enhancement Program for Bipolar Disorder (STEP-BD), a collaboration sponsored by the National Institute of Mental Health designed to evaluate the effectiveness of treatments for bipolar disorder. 366 patients were enrolled in this 26-week trial which included paroxetine up to $40 \mathrm{mg}$ /day, bupropion extended release up to $375 \mathrm{mg} /$ day added to lithium, valproate, carbamazepine or one of the approved antimanic agents. The results demonstrated that the use of antidepressant medication versus placebo adjunctive to mood stabilizers conferred no benefit. In addition the rates of treatmentemergent affective switch were similar in the antidepressant group and placebo. Although this study utilized only paroxetine and bupropion, which limits the generalizability to all other classes of antidepressants, it is the first largescale study designed to assess effectives of commonly employed antidepressants (paroxetine and bupropion) in the management of bipolar depression. Further long-term, head-to-head comparison studies are needed fully assess the role of antidepressants versus atypical antipsychotics in the management of bipolar depression.

\section{Conclusion}

At this stage, quetiapine appears to be the antipsychotic agent for which the evidence is strongest to support its use in the treatment of bipolar depression. When first published, initial findings from BOLDER I led clinicians to believe that the magnitude of antidepressant efficacy with quetiapine was large, though the confirmatory BOLDER II trial indicates that quetiapine's effects are likely to be only moderate. A post-hoc analysis (Cookson et al 2007) was recently published on BOLDER I data which suggested that when translated into clinically meaningful terms, the data indicate that between 4 and 9 patients would have to be treated with quetiapine in order for one additional patient to achieve a response beyond what would be produced by placebo. This illustrates the unmet need that still exists for effective and tolerable antidepressant agents in the management of bipolar depression. Given that quetiapine exerts both acute antidepressant and antimanic activity in monotherapy, it may be considered the atypical antipsychotic most representative of a "mood stabilizer". A shortcoming when considering the spectrum of quetiapine studies is the lack of maintenance phase data. Such studies would address whether antidepressant effects are maintained beyond the acute assessment period and address quetiapine's role in prevention of recurrence of new mood episodes, particularly new episodes of depression. Given the rather low response and remission rates with any agent in bipolar depression and the propensity for relapse, newer therapies continue to be needed to bring relief from this most complex illness.

\section{Disclosures}

Dr. Gajwani serves on speakerss bureaux for Forrest Pharmaceutical, Pfizer and Astra-Zeneca. He has received research grants from Otuska, Bristol Myers Squibb and Pfizer. He has served as consultant to Solvay Pharmaceuticals.

Dr. Muzina has served on speakers' bureaux and/or consulted for AstraZeneca, GlaxoSmithKline, and Pfizer and received research support through grants from Abbott Labs, GlaxoSmithKline, Eli Lilly Co, Novartis and Repligen.

Dr. Kemp has received educational grants from Abbott and Bristol-Myers Squibb and has received research support from GlaxoSmithKline.

Dr. Gao received grant support and/or honoraria from Abbott Laboratories, AstraZeneca, and GlaxoSmithKline

Dr. Calabrese has received Federal Funding from the Department of Defense, Health Resources Services Administration, National Institutes of Mental Health. Research or Grants from Private Industries or Non-Profit Funds from Cleveland Foundation, NARSAD, Repligen, Stanley Medical Research Institute. Advisory Boards served include Abbott, AstraZeneca, France Foundation, GlaxoSmithKline, Janssen, Johnson and Johnson Pharmaceutical Research and Development and Solvay/Wyeth pharmaceuticals. He has received Research Grants from Abbott, AstraZeneca, GSK, Janssen and Lilly. 


\section{References}

Altshuler LL, Gitlin MJ, Mintz J, et al. 2002. Subsyndromal depression is associated with functional impairment in patients with bipolar disorder. J Clin Psychiatry, 63:807-11.

Angst J. 1998. The emerging epidemiology of hypomania and bipolar II disorder. J Affect Disord, 50:143-51.

Calabrese JR, Hirschfeld RMA, Frye MA, et al. 2004. Impact of depressive symptoms as compared with manic symptoms in bipolar disorder: results of a US community-based sample. J Clin Psychiatry, 65:1499-504.

Calabrese JR, Keck PE, Macfadden W, et al. 2005. A randomized, doubleblind, placebo-controlled trial of quetiapine in the treatment of bipolar I and II depression. Am J Psychiatry, 162:1351-60.

Cookson J, Keck PE, Ketter TA, et al. 2007. Number needed to treat and time to response/remission for quetiapine monotherapy efficacy in acute bipolar depression: evidence from a large, randomized, placebocontrolled study. Int Clin Psychopharmacol, 22:93-100.

Gajwani P, Kemp DE, Muzina DJ, et al. 2006. Treatment of acute mania: An update on new medications. Current Psychiatric Reports, Bipolar Disorder, 8:504-9.

Goldstein J, Cristoph G, Grimm S, et al. 2007. Unique mechanism of action for antidepressant properties of the Atypical antipsychotic Quetiapine. Poster presented at APANR336:142.

Goodwin FK, Jamison KR. 1990. Manic-depressive illness. New York, NY: Oxford University Press.

Hamilton M. 1960. A rating scale for depression. J Neurol Neurosurg Psychiatry, 23:56-62.

Himmelhoch JM, Thase ME, allinger AG, et al. 1991. Tranylcypromine versus imipramine in anergic bipolar depression. Am J Psychiatry, 148:910-16.

Hlastala SA, Frank E, Malinger AG, et al. 1997. Bipolar depression: an underestimated treatment challenge. Depress Anxiety, 5:73-83.

Judd LL, Akiskal HS, Schetter PJ, et al. 2002. The long term natural history of the weekly symptomatic status of bipolar I disorder. Arch Gen Psychiatry, 59:503-37.
Judd LL, Akiskal HS, Schettler PJ, et al. 2003. A Prospective investigation of the natural history of the long-term weekly symptomatic status of bipolar II disorder. Arch Gen Psychiatry, 60:261-9.

Kessler RC, Chiu WT, Demler O, et al. 2005. Prevalence, severity, and comorbidity of 12-month DSM-IV disorders in the National Comorbidity Survey Replication. Arch Gen Psychiatry, 62:617-27.

Leverich GS, Altshuler LL, Frye MA, et al. 2003. Factors associated with suicide attempts in 648 patients with bipolar disorder in the stanley foundations bipolar network. J Clin Psychiatry, 64:506-15.

Leverich GS, Altshuler LL, Frye MA, et al. 2006. Risk of switch in mood polarity to hypomania or mania in patients with bipolar depression during acute and continuation trials of venlafaxine, sertaline, and bupropion as adjuncts to mood stabilizers. Am J Psychiatry, 163:232-9.

Montgomery SA, Åsberg M. 1979. A new depression scale designed to be sensitive to change. Br J Psychiatry, 134:382-9.

Muller MJ, Himmerich H, Kienzle B, et al. 2003. Differentiating moderate and severe depression using Montgomery-Åsberg depression rating scale (MADRS). J Affect Disord, 77:255-60.

Peet M. 1994. Induction of mania with selective serotonin re-uptake inhibitors and Tricyclic antidepressants. Br J Psychiatry, 164:549-50.

Post RM, Denicoff KD, Leverich GS, et al. 2003. Morbidity I 258 bipolar outpatients followed for 1 year with daily prospective rating on the NIMH life chart method. J Clin Psychiatry, 64:680-90.

Sachs GS, Nierenberg AA, Calabrese JR, et al. 2007. Effectiveness of adjunctive antidepressant treatment for bipolar depression. $N$ Eng $J$ Med, 356:1711-22.

Thase ME, Macfadden W, Weisler R, et al. 2006. Efficacy of quetiapine monotherapy in Bipolar I and II depression. J Clin Psychopharmacol, 26:600-9.

Tohen M, Vieta E, Calabrese JR, et al. 2003. Efficacy of olanzapine and olanzapine-fluoxetine combination in the treatment of bipolar I depression. Arch Gen Psychiatry, 60:1079-88.

Young RC, Biggs JT, Ziegler VE, et al. 1978. A rating scale for mania: reliability, validity and sensitivity. Br J Psychiatry, 133:429-35. 
